\title{
Unified Dual Mode Description of Small Molecule Sorption and Desorption Kinetics in a Glassy Polymer
}

\author{
Juchen Guo ${ }^{\dagger}$ and Timothy A. Barbari*, \\ ${ }^{\dagger}$ Department of Chemical and Biomolecular Engineering, University of Maryland, College Park, Maryland \\ 20742, and Department of Physics, Georgetown University, Washington, D.C. 20057
}

Received April 7, 2009; Revised Manuscript Received June 12, 2009

\begin{abstract}
FTIR-ATR (Fourier transform infrared-attenuated total reflectance) spectroscopy and a conventional gravimetric sorption balance were used to study sorption and desorption kinetics of vapor phase acetonitrile in glassy cellulose acetate. A time delay was observed in the measured concentration at the polymer-crystal interface from the FTIR-ATR experiments that was longer than expected from applying the simple Fickian diffusion model used to fit the gravimetric data for this system. By using the dual mode sorption model with total immobilization of the hole population rather than a simple Fickian framework, the time behavior at the boundary was readily captured. This model in turn was used to fit the FTIR-ATR data for desorption kinetics. The desorption diffusion coefficients were found to be considerably higher than those obtained for sorption owing to the necessary redistribution between the dissolved and hole populations when the classic dual mode sorption model is applied to the desorption isotherm. To resolve this inconsistency and remove the discontinuity in concentration at the penetrant-polymer boundary between sorption and desorption, a unified dual mode model to describe both sorption and desorption is proposed. In this model, a fraction of the dissolved population creates holes during the sorption process at each activity. The new model was applied to the sorption and desorption kinetics data in this study, resulting in unifying dual mode parameters and comparable diffusion coefficients.
\end{abstract}

\section{Introduction}

Understanding the diffusion of small molecules in glassy polymer films is important to a number of applications, including membrane separations, barrier materials, the controlled release of pharmaceuticals, and chemical sensors. Below the glasstransition temperature, large-scale molecular motions become severely hindered, and the resulting nonequilibrium nature of the material results in sorption and diffusion behavior that can be considerably more complicated than that in rubbery polymers, even when the diffusion mechanism is inherently Fickian. A number of techniques are available to determine the diffusion coefficient in a polymer. Film permeation methods are often employed at steady state to measure the molar flux across the membrane, from which the diffusion coefficient can be obtained provided that the equilibrium sorption has been measured, often gravimetrically. Gravimetric techniques are also used to measure sorption kinetics or the rate of mass uptake to determine the diffusion coefficient.

Time-resolved, Fourier transform infrared attenuated total reflectance (FTIR-ATR) spectroscopy has been used increasingly to study sorption kinetics in polymers and has proven to be very accurate and reliable. ${ }^{1}$ FTIR-ATR spectroscopy can provide reliable short-time data often missed with gravimetric techniques and is capable of identifying changes in the local environment for both the penetrant and the polymer, such as penetrant-penetrant and penetrant-polymer hydrogen bonding. Most of the previous studies using this spectroscopic approach have been with rubbery polymers because these materials adhere well to the ATR crystal, a requirement for obtaining reliable and reproducible data. One of the early studies from our group using FTIR-ATR spectroscopy was a comparison of the technique with gravimetry using the system methyl ethyl ketone in polyisobutylene. ${ }^{2} \mathrm{We}$ showed

*Corresponding author. E-mail: tab53@georgetown.edu. that the diffusion coefficients obtained from FTIR-ATR spectroscopy were well within experimental error of those measured gravimetrically. Put another way, which will be relevant to the current study, diffusion coefficients obtained spectroscopically could be used to predict gravimetric results and vice versa.

One of the unique features of FTIR-ATR spectroscopy applied to diffusion in polymer films is that it effectively measures the penetrant concentration at the polymer-crystal interface provided that the film thickness is at least 10 times greater than what is referred to as the depth of penetration of the ATR evanescent field into the polymer film. With film thicknesses on the order of $10 \mu \mathrm{m}$ and greater, this condition is readily met. In contrast, gravimetric techniques measure the total penetrant mass or the integrated average concentration in the film. These differences are depicted in Figure 1 , where $d_{\mathrm{p}}$ is the depth of penetration, $L$ is the film thickness, $A_{t}$ is the FTIR-ATR absorbance, $M_{t}$ is the mass uptake, and $C$ is the concentration. The present work was undertaken to test the robustness of sorption and transport models developed for glassy polymers by tracking the local concentration at the polymer-crystal interface during dynamic sorption and desorption. The system chosen for this study was acetonitrile in glassy cellulose acetate (CA), a polymer found to adhere well to the crystal with no evidence of delamination during sorption. The nitrile stretching band resides in a spectral region with little overlapping bands from the CA and likewise for the carbonyl stretching band in the $\mathrm{CA}$ with respect to the acetonitrile. Whereas the emphasis here is on the nitrile stretching band, changes in the carbonyl stretch can be used to monitor penetrant-induced swelling, ${ }^{3}$ which will be discussed in a subsequent paper for this system.

\section{Experimental Section}

Polymer Film Preparation. Acetonitrile and CA (39.8 wt \% acetyl content, average $M_{\mathrm{n}}$ ca. 30000$)$ were purchased from 

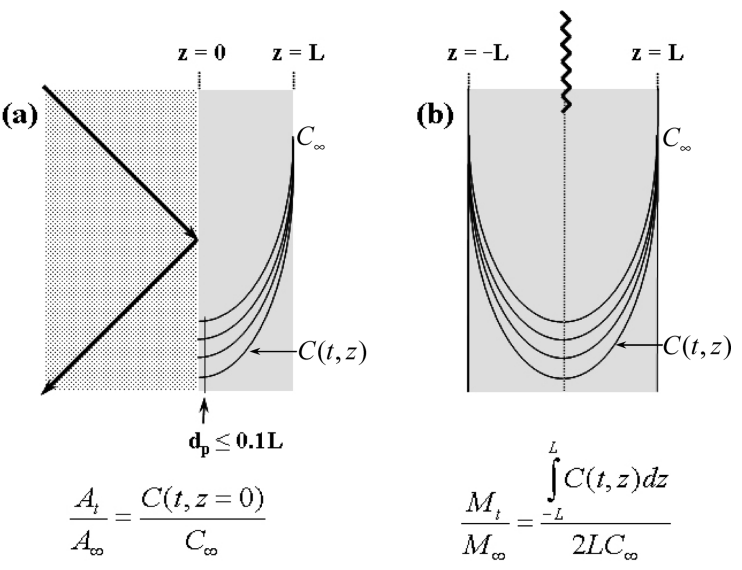

Figure 1. Comparison of concentration profiles from (a) FTIR-ATR spectroscopic and (b) gravimetric experiments to measure the diffusion coefficients. The normalized FTIR absorbance (a) is, in effect, the normalized local concentration at the polymer-crystal interface $(z=0)$. The normalized mass uptake (b) is a normalized average concentration.

Sigma-Aldrich and used as received. CA films were prepared by a solvent casting method. A solution of $3.9 \mathrm{wt} \% \mathrm{CA}$ in acetone (purchased from J. T. Baker, 99.5\%, used as received) was cast onto the appropriate substrate, either an ATR crystal or a glass coverslip, depending on whether a spectroscopic or gravimetric experiment, respectively, was to be performed. The substrate was placed in a fume hood for $24 \mathrm{~h}$ at room temperature, followed by another $24 \mathrm{~h}$ in a vacuum oven, preheated to $70{ }^{\circ} \mathrm{C}$. The substrate was then taken out of the oven and immediately placed in a fume hood at room temperature to cool. The dried film on the ATR crystal was found to contain no residual acetone by analysis of the polymer FTIR spectrum. Film thickness was measured using a micrometer $( \pm 2.5 \mu \mathrm{m})$.

Experimental Procedure. Gravimetry (quartz spring microbalance) was used to measure the equilibrium sorption isotherm for the system. The apparatus used for these measurements has been described elsewhere. ${ }^{4} \mathrm{CA}$ polymer films were prepared, as described above, on microscope glass coverslips $\left(2 \times 2 \mathrm{~cm}^{2}\right)$, removed intact, and hung directly on the quartz spring. The equilibrium mass uptake at a particular activity was determined from the spring extension, using a cathetometer, after $48 \mathrm{~h}$. Gravimetric sorption kinetics were monitored by tracking the extension in time for comparative purposes with results obtained spectroscopically.

Sorption kinetics experiments using FTIR-ATR spectroscopy were conducted at activities $0.06,0.1,0.15,0.2$, and 0.25 . The spectroscopic cell is connected to the same vapor generation system used for the gravimetric measurements, and the methods are described elsewhere. ${ }^{4}$ A background spectrum of the pure polymer on the ATR crystal was subtracted from all spectra obtained as a function of time during penetrant sorption and desorption. Penetrant activity was calculated from the ratio of the system pressure to the saturated vapor pressure of acetonitrile at the operating temperature of $25^{\circ} \mathrm{C}$. The Antoine equation was used to determine the saturated vapor pressure for acetonitrile. $^{5}$

Equilibrium desorption isotherms were measured gravimetrically at activities $0.15,0.2,0.25,0.3$, and 0.4 . To measure a desorption isotherm, a film was exposed to penetrant at the desired activity until equilibrium was reached. The activity was then decreased stepwise by applying vacuum to lower the system pressure, and the equilibrium mass uptake was recorded at each activity after $48 \mathrm{~h}$. FTIR-ATR desorption kinetics experiments were performed at the same activities used for sorption kinetics, after sorption equilibrium was approached, using the following procedure: (i) The ATR cell was isolated from the rest of the system by the closing of a valve. (ii) Vacuum was then applied to remove penetrant throughout the remainder of the system. (iii) When the pressure reached the lowest obtainable level (approximately $0.2 \mathrm{mmHg}$ ), the valve isolating the ATR cell was opened to vacuum, marking the starting time for desorption $(t=0)$.

Although the system was not at true vacuum, the boundary condition in mathematical descriptions of desorption was taken to be zero. Relative to the lowest equilibrium pressure prior to desorption $(5 \mathrm{mmHg}$ at activity 0.06 ), the assumption remains reasonable. Because the volume of the reservoir is much larger than that of the ATR cell, it is reasonable to assume that the surface of the CA film is exposed to vacuum immediately upon opening the connecting valve.

Analysis of Experimental Transport Data. To determine the diffusion coefficient from either the gravimetric or spectroscopic data, a transport model relating the mass uptake or infrared absorbance, respectively, to concentration $(C)$ is required. In the case of the gravimetric experiments, the relationship between mass uptake and concentration is straightforward and given by

$$
\frac{M_{t}}{M_{\infty}}=\frac{\int_{-L}^{L} C(t, z) \mathrm{d} z}{2 L C_{\infty}}
$$

where $M_{t}$ and $M_{\infty}$ are the sorbed masses at time $t$ and equilibrium, respectively, and $C_{\infty}$ is the sorbed concentration at equilibrium (also the boundary condition at $z= \pm L$ ). For the FTIR-ATR spectroscopic experiments, the equivalent relationship is more complicated owing to the exponential decay of the evanescent field into the polymer film and is given by ${ }^{1}$

$$
\frac{A_{t}}{A_{\infty}}=\frac{\int_{0}^{L} \exp \left(\frac{-2 z}{d_{\mathrm{p}}}\right) C(t, z) \mathrm{d} z}{\frac{d_{\mathrm{p}}}{2}\left[1-\exp \left(\frac{-2 L}{d_{\mathrm{p}}}\right)\right] C_{\infty}}
$$

If $d_{\mathrm{p}} \leq 0.1 L$ (thick-film approximation) and the concentration is relatively spatially invariant at $z<d_{\mathrm{p}}$ (no flux boundary condition), then eq 2 can be approximated by ${ }^{6}$

$$
\frac{A_{t}}{A_{\infty}}=\frac{C(t, z=0)}{C_{\infty}}
$$

In this study, the depth of penetration is approximately $1 \mu \mathrm{m}$, and the thickness of the CA film varies from 15 to $60 \mu \mathrm{m}$. Hence, the thick-film approximation is a reasonable assumption for this study.

\section{Transport Models}

Simple Fickian Diffusion. For 1D molecular diffusion in a polymer film, the governing equation for simple Fickian diffusion is

$$
\frac{\partial C}{\partial t}=\frac{\partial}{\partial z}\left(D \frac{\partial C}{\partial z}\right)
$$

where $D$ is the diffusion coefficient. Solving this equation with the boundary conditions shown in Figure 1 and an initial concentration of zero throughout the film results in a concentration profile, $C(t, z)$, that can be used with either eq 1 or eq 3 to obtain the measured mass uptake or IR absorbance, respectively, as a function of time. If the diffusion coefficient is taken to be constant (or concentration-averaged), then the mass uptake as a function of time from eq 1 is given by $^{7}$

$$
\frac{M_{t}}{M_{\infty}}=1-\sum_{n=0}^{\infty} \frac{8}{(2 n+1)^{2} \pi^{2}} \exp \left[\frac{-D(2 n+1)^{2} \pi^{2} t}{4 L^{2}}\right]
$$


The analogous expression for FTIR-ATR spectroscopy from eq 3 is ${ }^{6}$

$$
\begin{aligned}
\frac{A_{t}}{A_{\infty}} & =\frac{C(t, z=0)}{C_{\infty}} \\
& =1-\frac{4}{\pi} \sum_{n=0}^{\infty} \frac{(-1)^{n}}{2 n+1} \exp \left[\frac{-D(2 n+1)^{2} \pi^{2} t}{4 L^{2}}\right]
\end{aligned}
$$

Classic Dual Mode Sorption and Transport. For small molecules at low concentrations in glassy polymers, the most widely accepted model for sorption, the dual mode model, assumes that there are two populations of molecules in the polymer. One population, known as the Henry's law population, is dissolved in the dense portion of the polymer; the other one, known as the Langmuir population, resides in preexisting excess free volume or "holes". According to this model, the total concentration of sorbed penetrant is given by $^{8}$

$$
C=C_{\mathrm{D}}+C_{\mathrm{H}}=k_{\mathrm{D}} a+C_{\mathrm{H}}^{\prime} \frac{b a}{1+b a}
$$

where $k_{\mathrm{D}}$ is the Henry's law constant, $a$ is the penetrant activity in the gas or vapor phase, $\mathrm{C}^{\prime}{ }_{\mathrm{H}}$ is the Langmuir capacity, and $b$ is the Langmuir affinity constant.

The diffusion of small molecules in glassy polymers at low concentrations is typically represented as Fickian and assumes that the two penetrant species are mobile, with a fraction of the "hole" population immobilized. According to this partial immobilization hypothesis, a fraction, $F$, of the "hole" population, is mobile with the same diffusion coefficient as the "dissolved" population, represented by $D_{\mathrm{D}}$. Under these assumptions, species continuity equations can be written for each population. The most general form of the dual mode transport model is ${ }^{9}$

$$
\begin{gathered}
\frac{\partial C_{\mathrm{D}}}{\partial t}=\frac{\partial}{\partial z}\left(D_{\mathrm{D}} \frac{\partial C_{\mathrm{D}}}{\partial z}\right)-k_{\mathrm{f}} C_{\mathrm{D}}\left(C_{\mathrm{H}}^{\prime}-C_{\mathrm{H}}\right)+k_{\mathrm{r}} C_{\mathrm{H}} \\
\frac{\partial C_{\mathrm{H}}}{\partial t}=\frac{\partial}{\partial z}\left(F D_{\mathrm{D}} \frac{\partial C_{\mathrm{H}}}{\partial z}\right)+k_{\mathrm{f}} C_{\mathrm{D}}\left(C_{\mathrm{H}}^{\prime}-C_{\mathrm{H}}\right)-k_{\mathrm{r}} C_{\mathrm{H}}
\end{gathered}
$$

where $k_{\mathrm{f}}$ is the rate constant for hole filling and $k_{\mathrm{r}}$ is the rate constant for hole emptying.

Assuming local equilibrium between the two populations, the model can be reduced to

$$
\frac{\partial C}{\partial t}=\frac{\partial}{\partial z}\left(D_{\mathrm{D}} \frac{\partial C_{\mathrm{D}}}{\partial z}\right)+\frac{\partial}{\partial z}\left(F D_{\mathrm{D}} \frac{\partial C_{\mathrm{H}}}{\partial z}\right)
$$

with $C_{\mathrm{D}}$ and $C_{\mathrm{H}}$ related by

$$
k_{\mathrm{f}} C_{\mathrm{D}}\left(C_{\mathrm{H}}^{\prime}-C_{\mathrm{H}}\right)=k_{\mathrm{r}} C_{\mathrm{H}}
$$

Eliminating $C_{\mathrm{H}}$, the model for transport can be written for a constant or concentration-averaged $D_{\mathrm{D}}$ as

$$
\frac{\partial C}{\partial t}=D_{\mathrm{D}} \frac{\partial}{\partial z}\left[\frac{1+\frac{F K}{\left(1+\alpha C_{\mathrm{D}}\right)^{2}}}{\frac{\partial}{1+\frac{K}{\left(1+\alpha C_{\mathrm{D}}\right)^{2}}}} \frac{\partial C}{\partial z}\right]
$$

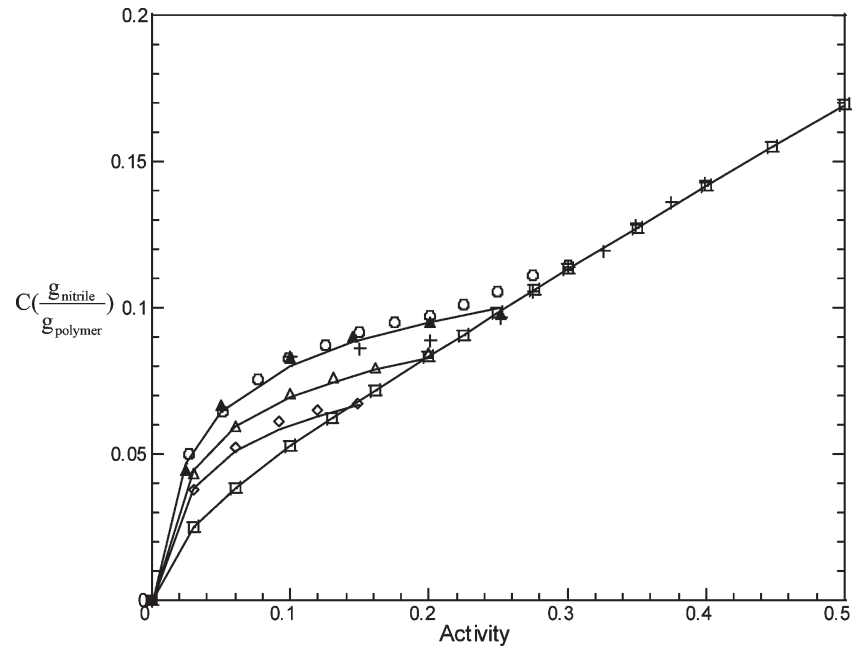

Figure 2. Acetonitrile sorption isotherm $(\square)$ and desorption isotherms at activities of $0.15(\diamond), 0.20(\triangle), 0.25(\mathbf{\Delta}), 0.30(\bigcirc)$, and $0.40(+)$ at $T=$ $25^{\circ} \mathrm{C}$. The solid lines represent dual mode model fits using eq 7 .

where

$$
\begin{gathered}
C_{\mathrm{D}}=\frac{-(1+K-\alpha C)+\left[(1+K-\alpha C)^{2}+4 \alpha C\right]^{1 / 2}}{2 \alpha} \\
\alpha=\frac{b}{k_{\mathrm{D}}} \\
K=\frac{C_{\mathrm{H}}^{\prime} b}{k_{\mathrm{D}}}=\alpha C_{\mathrm{H}}^{\prime}
\end{gathered}
$$

The initial condition and boundary conditions for sorption are

$$
\begin{gathered}
t=0(\text { all } z) \quad C=0 \\
z=0(t>0) \quad \frac{\partial C}{\partial z}=0 \\
z=L(t>0) \quad C=C_{\infty}
\end{gathered}
$$

For desorption, the initial condition and boundary conditions are

$$
\begin{gathered}
t=0(\text { all } z) \quad C=C_{\infty} \\
z=0(t>0) \quad \frac{\partial C}{\partial z}=0 \\
z=L(t>0) \quad C=0
\end{gathered}
$$

\section{Results and Discussion}

Sorption-Desorption Hysteresis for Acetonitrile in Cellulose Acetate. The sorption isotherm for acetonitrile in CA is plotted in Figure 2 as a function of activity. The dual mode sorption model, eq 7, was used to fit the data to obtain the 
Table 1. Dual Mode Parameters for Desorption of Acetonitrile in CA at Various Activities at $T=25^{\circ} \mathrm{C} . b=38.1$ from Sorption Isotherm

\begin{tabular}{cccc}
\hline & & \multicolumn{2}{c}{$C_{\mathrm{Hd}}^{\prime}\left(g_{\text {nitrile }} / g_{\text {polymer }}\right)$} \\
\cline { 3 - 4 } activity & $k_{\text {Dd }}\left(g_{\text {nitrile }} / g_{\text {polymer }}\right)$ & fit & calculated $^{b}$ \\
\hline 0.25 & 0.0585 & 0.0944 & 0.0915 \\
0.20 & 0.0602 & 0.0834 & 0.0804 \\
0.15 & 0.0592 & 0.0715 & 0.0693 \\
0.10 & $0.0593^{a}$ & & 0.0581 \\
0.06 & $0.0593^{a}$ & & 0.0492 \\
${ }^{a}$ Average value from activities $0.25,0.2$, and $0.15 .{ }^{b}$ From eq 18.
\end{tabular}

dual mode sorption parameters

$$
\begin{gathered}
k_{\mathrm{D}}=0.282 \frac{g_{\text {nitrile }}}{g_{\text {polymer }}} \\
C_{\mathrm{H}}^{\prime}=0.030 \frac{g_{\text {nitrile }}}{g_{\text {polymer }}} \\
b=38.1
\end{gathered}
$$

It is well known that the sorption and transport properties of glassy polymers are history-dependent. ${ }^{10-14}$ Sorptiondesorption hysteresis is often observed for small molecule isotherms in glassy polymers. Such an effect was observed in the acetonitrile/CA system. Desorption isotherms were measured at three different activities, 0.15, 0.20, and 0.25. The results are plotted in Figure 2 along with the fits using eq 7. In applying eq 7 to the desorption data, the "hole" affinity constant, $b$, was assumed to be the same as that for sorption and hence was fixed at 38.1. The adjustable desorption dual mode parameters, $k_{\mathrm{Dd}}$ and $C_{\mathrm{Hd}}$, are listed in Table 1 . The value of the Henry's law constant from the desorption isotherms is essentially constant at $k_{\mathrm{Dd}}=(0.0593 \pm 0.0008)$ $\left(g_{\text {nitrile }} / g_{\text {polymer }}\right)$ for the three activities.

Desorption isotherms were also measured for activities 0.30 and 0.40 . The desorption isotherm starting from activity 0.4 follows the sorption isotherm initially and then departs below activity 0.25 . Similarly, the desorption isotherm starting from activity 0.3 departs from the sorption isotherm near activity 0.25 . Ultimately, both follow the desorption isotherm generated from activity 0.25 . These results strongly suggest that the CA is fully relaxed above activity 0.25 . Hence, a glass transition induced by acetonitrile was taken to be at activity 0.25 , where $C_{\mathrm{g}}=0.0977\left(g_{\text {nitrile }} / g_{\text {polymer }}\right)$.

Sorption Kinetics. Gravimetric measurements were performed at activities of $0.06,0.1$ and 0.2 , and the data were fit using eq 5. A representative example of mass uptake data at activity 0.2 and the corresponding fit are shown in Figure 3. The linear dependence at short times between mass uptake and the square root of time clearly shows that the underlying physical mechanism for diffusion in the acetonitrile-CA system is Fickian. Experiments at each activity were done in triplicate. The diffusion coefficients are listed in Table 2. A representative experiment using FTIR-ATR is shown in Figure 4 , also at activity 0.2. Equation 6 was used to calculate the concentration at the polymer-crystal interface as a function of time using the average diffusion coefficient obtained from mass uptake at the same activity, $D=6.5 \times$ $10^{-10} \mathrm{~cm}^{2} / \mathrm{s}$. The resulting prediction shown in Figure 4a is not able to capture the data, particularly at short times. Equation 6 was also used to obtain the best fit of the data, resulting in a diffusion coefficient of $6.25 \times 10^{-10} \mathrm{~cm}^{2} / \mathrm{s}$ for this particular experiment. However, the fit is rather poor, as

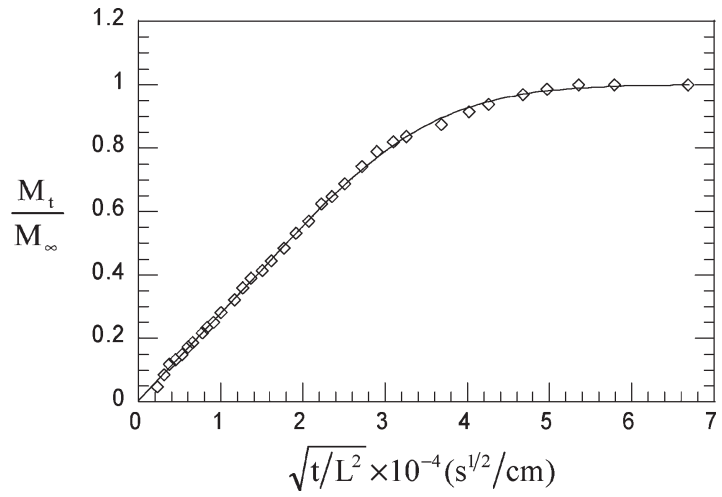

Figure 3. Representative mass uptake kinetics and the fit using eq 5 at activity $0.2 . L=50.4 \mu \mathrm{m}$ and $T=25^{\circ} \mathrm{C}$.

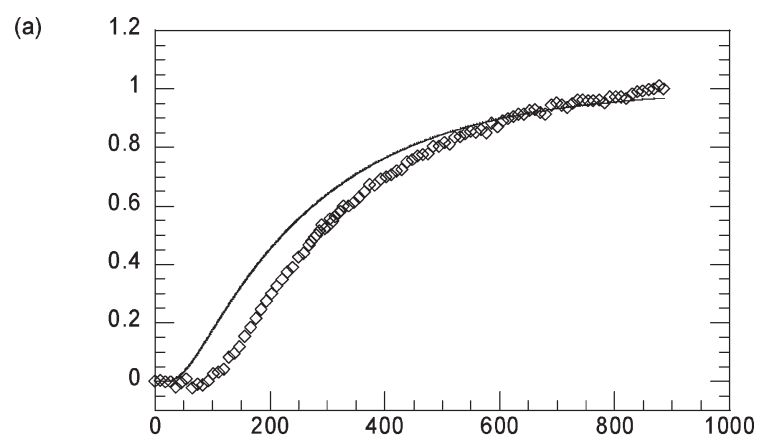

$\frac{\mathrm{C}_{\mathrm{t}, \mathrm{z}=0}}{\mathrm{C}_{\infty}}$

(b)

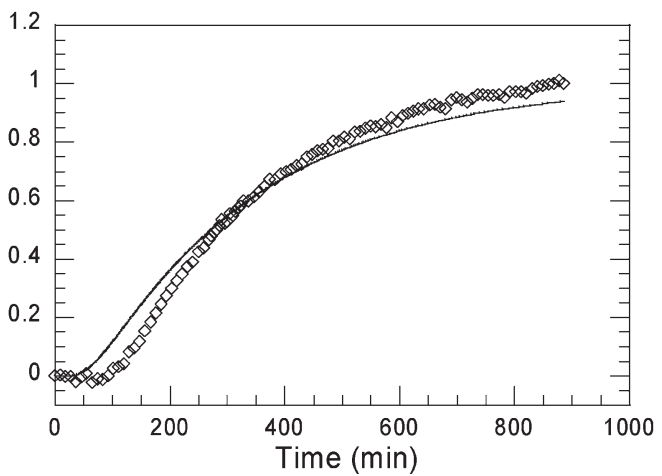

Figure 4. Representative FTIR-ATR absorbance data of acetonitrile in CA at activity 0.2 with (a) the calculated normalized concentration using the simple Fickian model, eq 6 , and the average diffusion coefficient obtained from the gravimetric measurements and (b) the best fit using eq 6. $L=49.2 \mu \mathrm{m}$ and $T=25^{\circ} \mathrm{C}$.

shown in Figure 4b. It is clear that the time delay in the spectroscopic data is longer than expected and cannot be captured by a simple Fickian model. This is in contrast with rubbery polymers in which the time delay is readily captured, and a simple Fickian model can describe both gravimetric and spectroscopic data well with the same value of $D .^{2}$

Using the dual mode sorption parameters, the corresponding transport model, eq 12, was used to fit the FTIR-ATR absorbance data for sorption kinetics from triplicate experiments, using $D_{\mathrm{D}}$ as the only adjustable parameter and $F$ set to zero. The diffusion coefficients so obtained are listed in Table 2. A representative fit of the sorption kinetics at activity 0.2 is shown in Figure 5, with $D=6.34 \times 10^{-10} \mathrm{~cm}^{2} / \mathrm{s}$. Whereas steady-state transport experiments (permeability measurements) as a function of activity (or pressure) can be described with unique values of $D_{\mathrm{D}}$ and $F$, setting 
Table 2. Diffusion Coefficients for Sorption and Desorption for Acetonitrile in $\mathrm{CA}$ at $25^{\circ} \mathrm{C}$

\begin{tabular}{cllll}
\hline & \multicolumn{3}{c}{$D_{\mathrm{D}} \times 10^{9} \mathrm{~cm}^{2} / \mathrm{s}$} \\
\cline { 2 - 5 } activity & sorption from mass uptake & sorption from classic dual mode & sorption from dual mode with hole formation & desorption \\
\hline 0.06 & $0.21 \pm 0.03$ & $0.19 \pm 0.01$ & $0.90 \pm 0.05$ & $0.95 \pm 0.02$ \\
0.10 & $0.32 \pm 0.04$ & $0.29 \pm 0.01$ & $1.43 \pm 0.06$ & $1.82 \pm 0.35$ \\
0.15 & & $0.46 \pm 0.02$ & $2.21 \pm 0.12$ & $3.45 \pm 0.005$ \\
0.20 & $0.65 \pm 0.04$ & $0.65 \pm 0.02$ & $3.12 \pm 0.08$ & $6.70 \pm 0.74$ \\
0.25 & & $0.74 \pm 0.09$ & $3.54 \pm 0.42$ & $10.44 \pm 1.06$
\end{tabular}

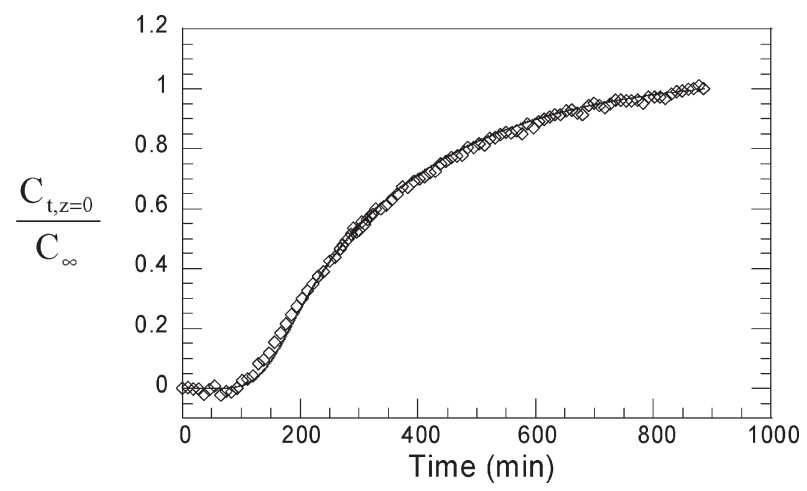

Figure 5. Representative FTIR-ATR absorbance data of acetonitrile in CA at activity 0.2 using the dual mode transport model, eq 12 , and sorption parameters from the classic dual mode sorption model, eq 7. $L=49.2 \mu \mathrm{m}$ and $T=25^{\circ} \mathrm{C}$.

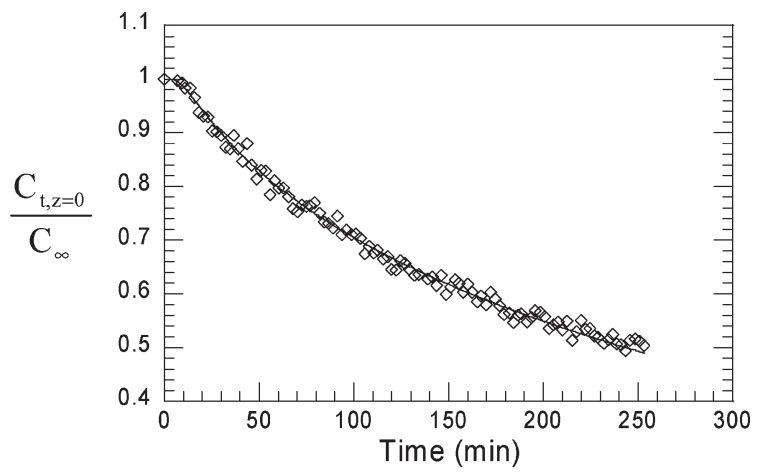

Figure 6. Representative FTIR-ATR desorption kinetics for acetonitrile in CA at activity 0.15 and the fit using eq 12 with dual mode parameters from fitting the desorption isotherm at 0.15. (See Table 1.) $L=29.2 \mu \mathrm{m}$ and $T=25^{\circ} \mathrm{C}$.

$F$ equal to physically realistic values of 0.1 or 0.2 only serves to shift the value of each newly fit $D_{\mathrm{D}}$ at each activity by an equivalent amount. Therefore, total immobilization of the "hole" population was assumed for all subsequent analyses.

Desorption Kinetics Using the Classic Dual Mode Model. The dual mode desorption parameters, $k_{\mathrm{Dd}}$ and $C_{\mathrm{Hd}}^{\prime}$ in Table 1 were used to fit the desorption kinetics data from FTIR-ATR spectroscopy at activities $0.15,0.2$, and 0.25 using eq 12 with $D_{\mathrm{D}}$ as the only adjustable parameter $(F=0)$. The model captures the data well, and representative fits are plotted in Figures 6-8. From triplicate experiments, mean values of $D_{\mathrm{D}}$ were determined at each activity and are listed in Table 2 for comparison with those obtained from sorption kinetics. From Table 2, it can be seen that the diffusion coefficients for sorption and desorption at a given activity differ considerably. Whereas polymer chain mobility certainly is affected by the presence of penetrant molecules, it seems highly unrealistic that such changes would manifest themselves in such large differences in diffusion coefficients for desorption kinetics relative to sorption kinetics. A closer

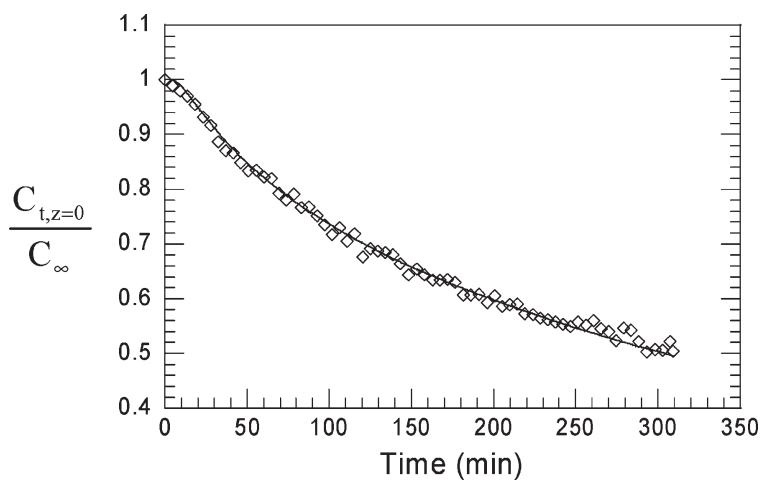

Figure 7. Representative FTIR-ATR desorption kinetics for acetonitrile in CA at activity 0.20 and the fit using eq 12 with dual mode parameters from fitting the desorption isotherm at 0.20. (See Table 1.). $L=44.0 \mu \mathrm{m}$ and $T=25^{\circ} \mathrm{C}$.

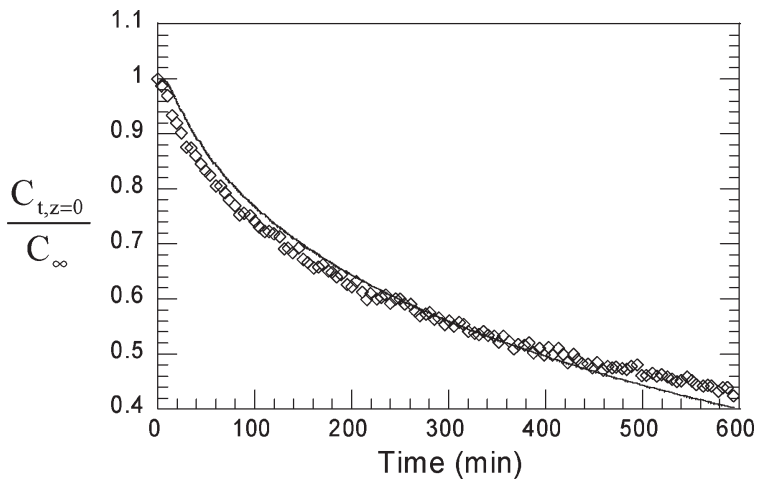

Figure 8. Representative FTIR-ATR desorption kinetics for acetonitrile in CA at activity 0.25 and the fit using eq 12 with dual mode parameters from fitting the desorption isotherm at 0.25 . (See Table 1.) $L=60.8 \mu \mathrm{m}$ and $T=25^{\circ} \mathrm{C}$.

examination of the classic dual mode model reveals that the penetrant populations must be dramatically redistributed at the start of desorption to obtain these results.

As the dual mode parameters for the desorption isotherms in Table 1 suggest, desorption hysteresis distinguishes itself from sorption by significantly higher values of $C_{\mathrm{Hd}}^{\prime}$, reflecting a redistribution of the penetrant population from $C_{\mathrm{D}}$ to $C_{\mathrm{H}}$. In other words, a portion of the penetrant molecules that were counted as part of the dissolved population during sorption must be counted as part of the Langmuir population upon desorption. In Figure 9, calculated values of the total concentration, the Henry's law population, and the Langmuir population at the polymer-crystal interface for sorption and desorption kinetics at activity 0.2 are plotted. This figure clearly shows that there must be an instantaneous increase in the Langmuir population and concomitant decrease in the Henry's law population at the start of desorption if the classic dual mode model is to be followed. This sudden redistribution between the two populations is not readily interpretable physically. 


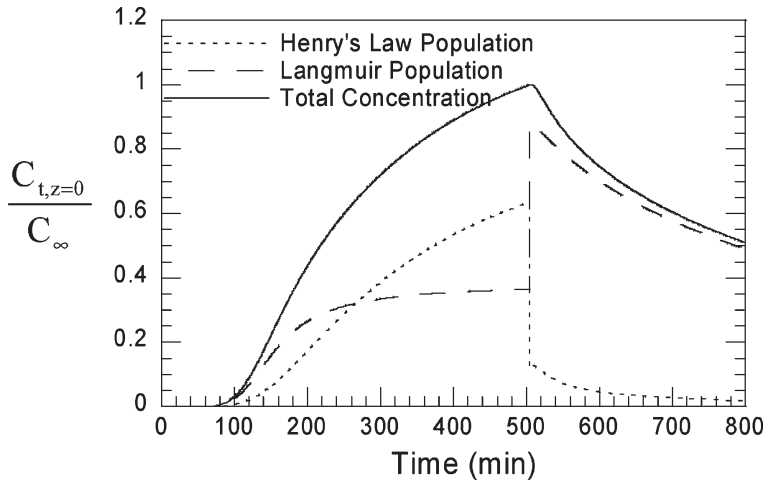

Figure 9. Penetrant population distribution from classic dual mode model as a function of time for sorption and desorption of acetonitrile in CA. Calculated from experiment at activity 0.20. $L=44.0 \mu \mathrm{m}$ and $T=$ $25^{\circ} \mathrm{C}$.
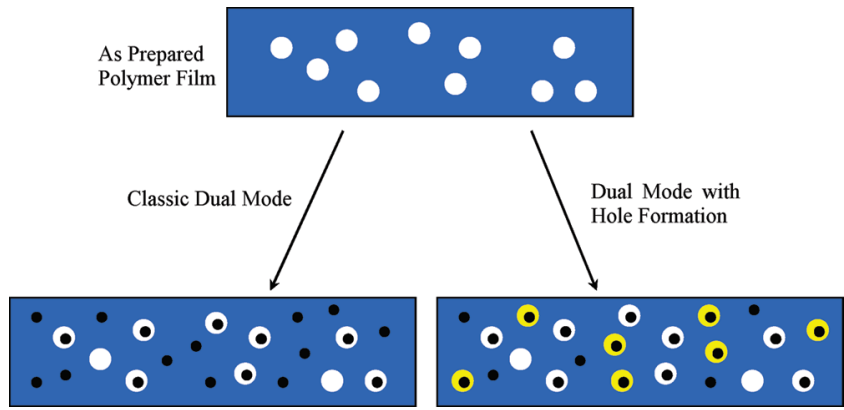

Figure 10. Schematic comparison of classic dual mode sorption and dual mode with hole formation at constant $C$.

Unified Dual Mode Model for Sorption and Desorption. The sorption-desorption hysteresis shown in Figure 2 suggests that the physical structure of the CA polymer changes during the penetrant sorption process. The increasing values of $C_{\mathrm{Hd}}^{\prime}$ for the desorption isotherms with increasing initial activity confirm this. Hence, one could envision that the Langmuir capacity may not be constant with activity during sorption kinetics and that additional holes are created by a portion of the Henry's law population continuously at any position within the film. Physically, thermal fluctuations in the polymer chains must create enough local free volume for a penetrant molecule to initiate a diffusion step, which is only successful if the chains close behind the penetrant molecule. If the chains do not close, then the penetrant molecule "falls back" and the diffusion jump is unsuccessful. This particular diffusing molecule becomes trapped in a low energy site that it created, resulting in a higher energy barrier for the molecule to leave. In other words, what was a "dissolved" molecule creates a new "hole" and becomes reassigned to the hole population. When this molecule overcomes the high energy barrier and leaves the hole, the hole remains and increases the Langmuir capacity. This hole forming process is schematically shown in Figure 10.

To describe hole formation mathematically, assume that at any given activity the total penetrant concentration can be expressed as

$$
C=C_{\mathrm{D}}^{*}+C_{\mathrm{H}}^{*}
$$

where $C_{\mathrm{D}}^{*}=\left(1-f_{\mathrm{H}}\right) C_{\mathrm{D}} ; C_{\mathrm{H}}^{*}=C_{\mathrm{H}}+f_{\mathrm{H}} C_{\mathrm{D}}$, and $f_{\mathrm{H}}$ is the fraction of dissolved molecules during sorption in the classic dual mode framework that form new holes. $C_{\mathrm{H}}$ and $C_{\mathrm{D}}$ in that framework are related by

$$
C_{\mathrm{H}}=C_{\mathrm{H} 0}^{\prime} \frac{\alpha C_{\mathrm{D}}}{1+\alpha C_{\mathrm{D}}}
$$

where $C_{\mathrm{H} 0}^{\prime}$ is the pre-existing Langmuir capacity for the pure, as-prepared polymer and $\alpha=b / k_{\mathrm{D}}$. The total penetrant concentration, after rearrangement, is

$$
C=\left(1-f_{\mathrm{H}}\right) C_{\mathrm{D}}+C_{\mathrm{H} 0}^{\prime} \frac{\left[1+\frac{f_{\mathrm{H}}}{\alpha C_{\mathrm{H} 0}^{\prime}}\left(1+\alpha C_{\mathrm{D}}\right)\right] \alpha C_{\mathrm{D}}}{1+\alpha C_{\mathrm{D}}}
$$

In terms of activity, using $C_{\mathrm{D}}=k_{\mathrm{D}} a$

$$
C=\left(1-f_{\mathrm{H}}\right) k_{\mathrm{D}} a+C_{\mathrm{H} 0}^{\prime}\left(1+\eta \frac{1+b a}{b}\right)\left(\frac{b a}{1+b a}\right)
$$

where $\eta=\frac{f_{\mathrm{H}} k_{\mathrm{D}}}{C^{\prime}}$. In eq 16 , the Langmuir capacity during sorption increases with activity according to

$$
C_{\mathrm{H}}^{\prime}=C_{\mathrm{H} 0}^{\prime}\left(1+\eta \frac{1+b a}{b}\right)
$$

Equation 16 is a form of the dual mode sorption model that accounts for hole formation with $k_{\mathrm{D}}=0.282\left(g_{\text {nitrile }} / g_{\text {polymer }}\right)$, $b=38.1$, and $C_{\mathrm{H} 0}^{\prime}=0.030\left(g_{\text {nitrile }} / g_{\text {polymer }}\right)$ (the same values in the classic dual mode model for sorption).

During the desorption process, if the penetrant molecules comprising the Langmuir population leave the holes and the holes do not close behind them, then the Langmuir capacity at a particular starting activity $C_{\mathrm{Hd}}^{\prime}$ remains unchanged and is given by

$$
C_{\mathrm{Hd}}^{\prime}=C_{\mathrm{H} 0}^{\prime}\left(1+\eta \frac{1+b a_{\mathrm{d}}}{b}\right)=C_{\mathrm{H} 0}^{\prime}+f_{\mathrm{H}} k_{\mathrm{D}} \frac{1+b a_{\mathrm{d}}}{b}
$$

In Equation 18, $a_{\mathrm{d}}$ is fixed at the activity at the start of desorption. As a result, the classic form of the dual mode model is recaptured for desorption

$$
C=k_{\mathrm{Dd}} a+C_{\mathrm{Hd}}^{\prime} \frac{b a}{1+b a}
$$

where $k_{\mathrm{Dd}}=\left(1-f_{\mathrm{H}}\right) k_{\mathrm{D}}$. As shown in Table 1 , fitting the desorption isotherms at activities $0.15,0.2$, and 0.25 resulted in an average value of $k_{\mathrm{Dd}}=0.0593\left(g_{\text {nitrile }} / g_{\text {polymer }}\right)$. Therefore, $f_{\mathrm{H}}$ can be calculated to be 0.79 for the $\mathrm{CA}$-acetonitrile system here.

Using the values of $C_{\mathrm{H} 0}^{\prime}, k_{\mathrm{D}}$, and $f_{\mathrm{H}}$ previously determined, the Langmuir capacities for desorption at activities 0.06 and 0.1 , which were not obtained experimentally from desorption isotherms, can be calculated using eq 18. The calculated values at all activities are listed in Table 1 . Those at activities of $0.15,0.2$, and 0.25 are in excellent agreement with those fit from the classic dual mode model. With the calculated Langmuir capacities, desorption kinetics at activities 0.06 and 0.1 were fit with $D_{\mathrm{D}}$ as the only adjustable parameter. Representative fits are shown in Figures 11 and 12, respectively.

Sorption Kinetics Using the Dual Mode Model with Hole Formation. The resulting transport model based on $C_{\mathrm{D}}^{*}$ and 


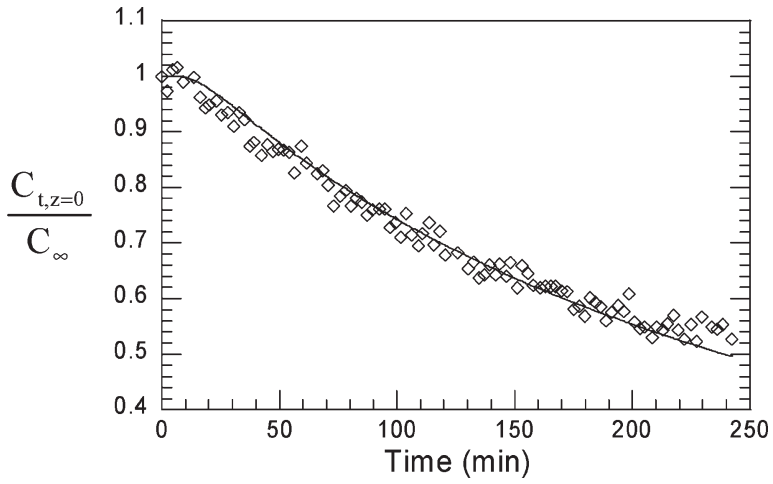

Figure 11. Representative FTIR-ATR desorption kinetics for acetonitrile in CA at activity 0.06 and the fit using eq 12 with the calculated value of $C_{\mathrm{Hd}}^{\prime}$. (See Table 1.) $L=15.2 \mu \mathrm{m}$ and $T=25^{\circ} \mathrm{C}$.

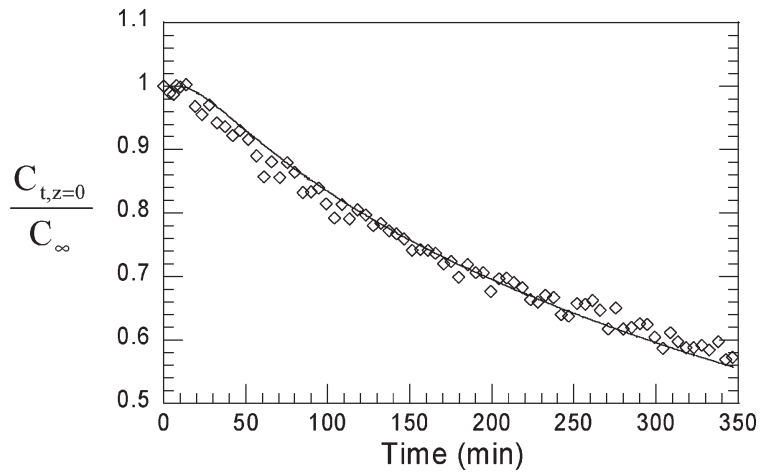

Figure 12. Representative FTIR-ATR desorption kinetics for acetonitrile in CA at activity 0.10 and the fit using eq 12 with the calculated value of $C_{\mathrm{Hd}}^{\prime}$. (See Table 1.) $L=29.8 \mu \mathrm{m}$ and $T=25^{\circ} \mathrm{C}$.

$C_{\mathrm{H}}^{*}$ for sorption kinetics is

$$
\begin{gathered}
\frac{\partial C_{\mathrm{D}}^{*}}{\partial t}=\frac{\partial}{\partial z}\left(D_{\mathrm{D}} \frac{\partial C_{\mathrm{D}}^{*}}{\partial z}\right) \\
-k_{\mathrm{f}} C_{\mathrm{D}}^{*}\left(C_{\mathrm{H} 0}^{\prime}+\frac{f_{\mathrm{H}}}{1-f_{\mathrm{H}}} C_{\mathrm{D}}^{*}+\frac{f_{\mathrm{H}}}{\alpha}-C_{\mathrm{H}}^{*}\right)+k_{\mathrm{r}} C_{\mathrm{H}}^{*} \\
\frac{\partial C_{\mathrm{H}}^{*}}{\partial t}=\frac{\partial}{\partial z}\left(F D_{\mathrm{D}} \frac{\partial C_{\mathrm{H}}^{*}}{\partial z}\right) \\
+k_{\mathrm{f}} C_{\mathrm{D}}^{*}\left(C_{\mathrm{H} 0}^{\prime}+\frac{f_{\mathrm{H}}}{1-f_{\mathrm{H}}} C_{\mathrm{D}}^{*}+\frac{f_{\mathrm{H}}}{\alpha}-C_{\mathrm{H}}^{*}\right)-k_{\mathrm{r}} C_{\mathrm{H}}^{*}
\end{gathered}
$$

Assuming local equilibrium between the two populations and taking $D_{\mathrm{D}}$ to be either constant or a concentration-averaged value at a given activity, the model can be reduced to

$$
\frac{\partial C}{\partial t}=D_{\mathrm{D}} \frac{\partial}{\partial z}\left[\frac{\frac{1+F\left(S+2 \delta C_{\mathrm{D}}^{*}+\beta \delta C_{\mathrm{D}}^{* 2}\right)}{\left(1+\beta C_{\mathrm{D}}^{*}\right)^{2}}}{\frac{1+S+2 \delta C_{\mathrm{D}}^{*}+\beta \delta C_{\mathrm{D}}^{* 2}}{\left(1+\beta C_{\mathrm{D}}^{*}\right)^{2}}} \frac{\partial C}{\partial z}\right]
$$

where

$$
\begin{gathered}
C_{\mathrm{D}}^{*}=\frac{-(1+S-\beta C)+\left[(1+S-\beta C)^{2}+4(\beta+\delta) C\right]^{1 / 2}}{2(\beta+\delta)} \\
\beta=\frac{b}{\left(1-f_{\mathrm{H}}\right) k_{\mathrm{D}}}
\end{gathered}
$$

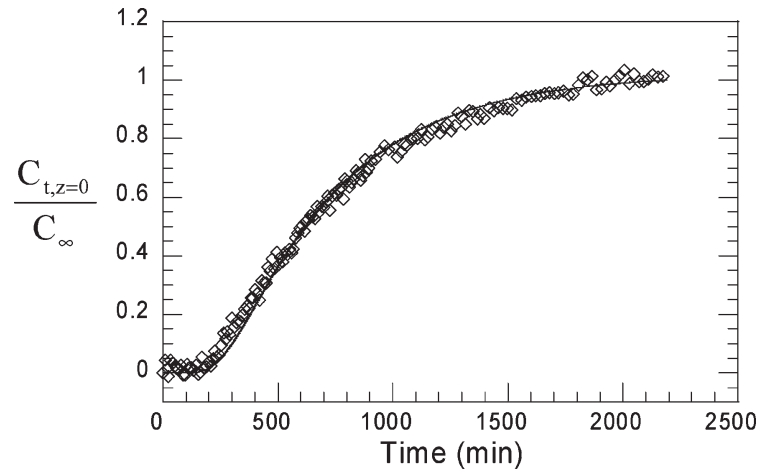

Figure 13. Representative FTIR-ATR sorption kinetics for acetonitrile in CA at activity 0.06 using dual mode sorption with hole formation, eq 22. $L=20.8 \mu \mathrm{m}$ and $T=25^{\circ} \mathrm{C}$.

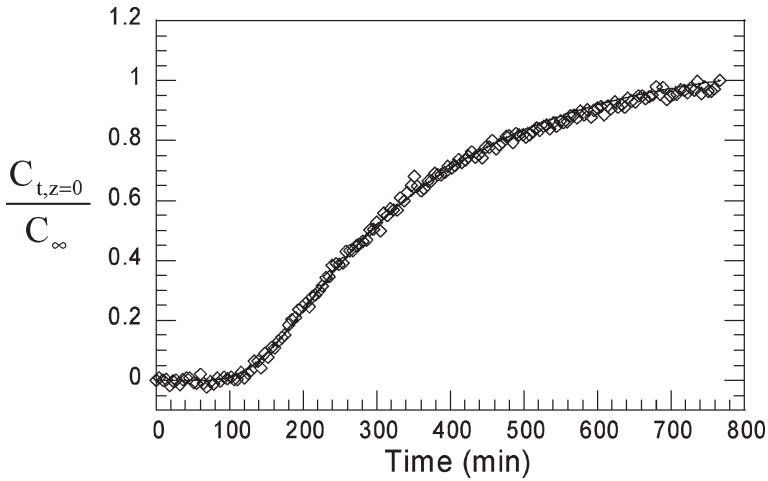

Figure 14. Representative FTIR-ATR sorption kinetics for acetonitrile in CA at activity 0.10 using dual mode sorption with hole formation, eq 22. $L=29.8 \mu \mathrm{m}$ and $T=25^{\circ} \mathrm{C}$.

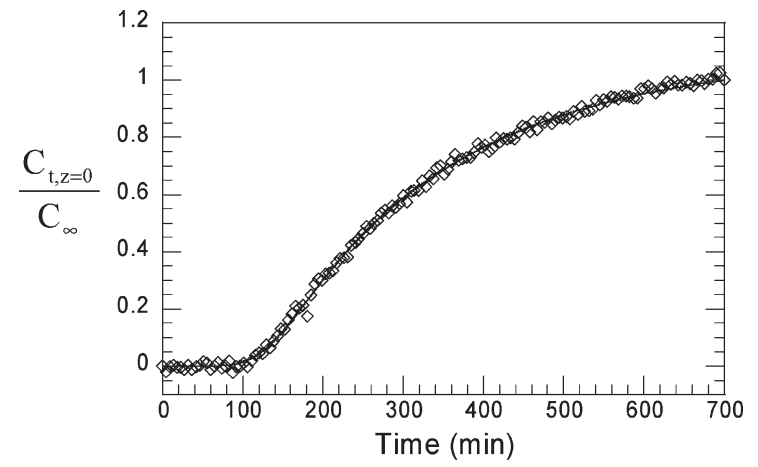

Figure 15. Representative FTIR-ATR sorption kinetics for acetonitrile in CA at activity 0.15 using dual mode sorption with hole formation, eq 22. $L=38.4 \mu \mathrm{m}$ and $T=25^{\circ} \mathrm{C}$.

$$
\begin{gathered}
S=\beta\left(C_{\mathrm{H} 0}^{\prime}+\frac{f_{\mathrm{H}}}{\alpha}\right) \\
\delta=\beta \frac{f_{\mathrm{H}}}{1-f_{\mathrm{H}}}
\end{gathered}
$$

The sorption kinetics were fit using eq 22 with the following parameters fixed: $k_{\mathrm{D}}=0.282\left(g_{\text {nitrile }} / g_{\text {polymer }}\right), C_{\mathrm{H} 0}^{\prime}=0.030$ $\left(g_{\text {nitrile }} / g_{\text {polymer }}\right), f_{\mathrm{H}}=0.79$, and $b=38.1$. The diffusion coefficient, $D_{\mathrm{D}}$, is the only adjustable parameter assuming $F=0$. Representative fits at each of the activities are shown in Figures 13-17, and the mean values of $D_{\mathrm{D}}$ from triplicate experiments are listed in Table 2. From Table 2, it can be seen 


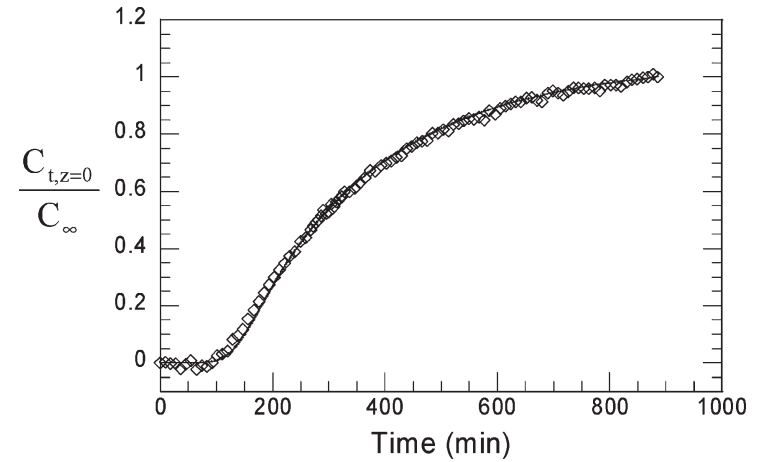

Figure 16. Representative FTIR-ATR sorption kinetics for acetonitrile in CA at activity 0.20 using dual mode sorption with hole formation, eq 22. $L=49.2 \mu \mathrm{m}$ and $T=25^{\circ} \mathrm{C}$.

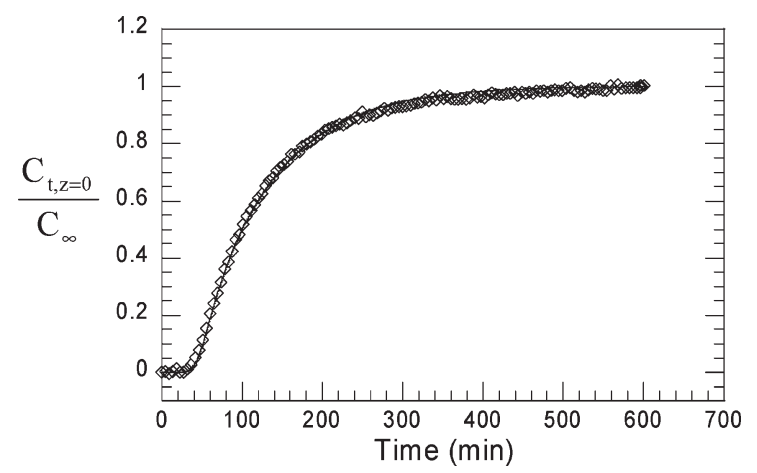

Figure 17. Representative FTIR-ATR sorption kinetics for acetonitrile in CA at activity 0.25 using dual mode sorption with hole formation, eq 22. $L=30.4 \mu \mathrm{m}$ and $T=25^{\circ} \mathrm{C}$.

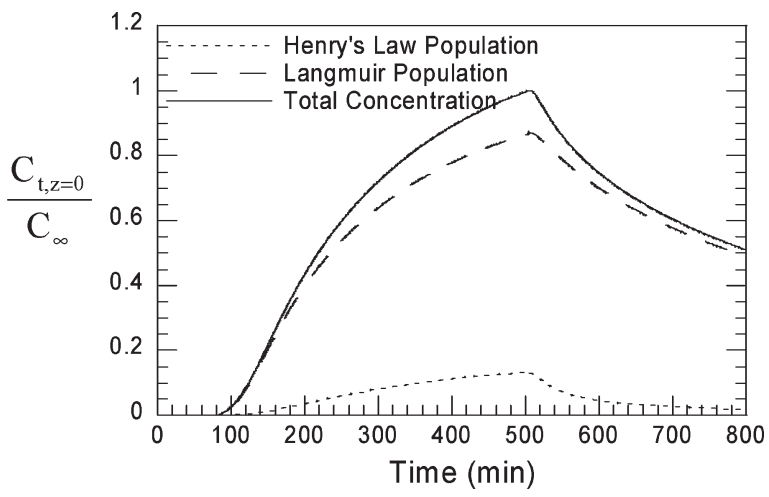

Figure 18. Penetrant population distribution from dual mode model with hole formation as a function of time for sorption and desorption of acetonitrile in CA. Calculated from experiment at activity $0.20 . L=$ $44.0 \mu \mathrm{m}$ and $T=25^{\circ} \mathrm{C}$.

that the diffusion coefficients for sorption and desorption from the dual mode model with hole formation are in much better agreement. Using this unified dual mode description, the total concentration, the Henry's law population, and the Langmuir population at the polymer-crystal interface during sorption and desorption at activity 0.2 were calculated and plotted in Figure 18. The diffusing "dissolved" population is much lower during sorption with hole formation, which results in a higher diffusion coefficient to match the data. Furthermore, the redistribution between populations occurs continuously during sorption, eliminating the discontinuity at the start of desorption.

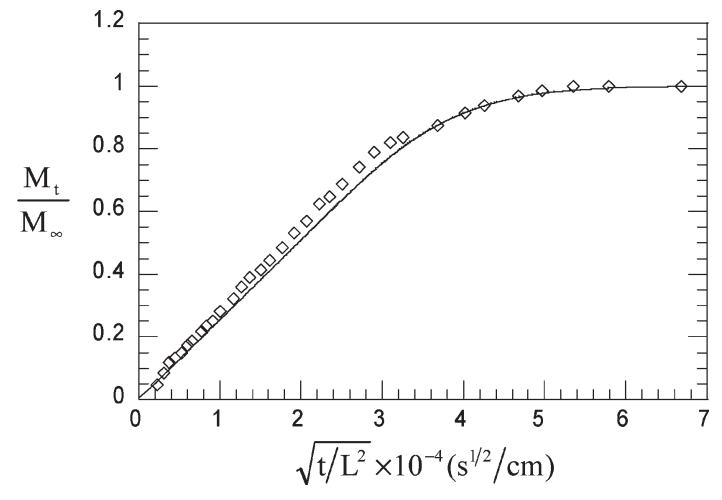

Figure 19. Prediction of mass uptake at activity 0.2 using the dual mode sorption model with hole formation. Open diamonds represent mass uptake data. $L=50.4 \mu \mathrm{m}$ and $T=25^{\circ} \mathrm{C}$.

Prediction of Mass Uptake. As a further validation of this unified approach, the mass uptake was predicted using the parameters obtained from the dual mode sorption model with hole formation. First, the total concentration profile was calculated numerically, and then, this profile was integrated over the film thickness to obtain the mass uptake. (See Figure 1.) The prediction uses the average diffusion coefficient from the dual mode model with hole formation. A representative example for activity 0.2 along with the actual mass uptake data is plotted as a function of $\left(t / \mathrm{L}^{2}\right)^{1 / 2}$ in Figure 19. In general, there is good agreement between the prediction and the actual data. Similar results were obtained at the other activities.

\section{Conclusions}

A simple Fickian diffusion model was unable to capture local concentration data in time for sorption kinetics, as measured by FTIR-ATR spectroscopy, but the classic dual mode sorption model with total immobilization of the hole population fit the data well. The resulting concentration-averaged diffusion coefficients themselves increase with increasing activity, reflecting an increase in chain mobility (plasticization) with concentration. When applied to desorption kinetics, excellent fits were again obtained but with diffusion coefficients that were considerably higher than those for sorption. This can be explained by the discontinuity in both the dissolved and hole populations between the end of sorption and the start of desorption, which is imposed by the classic dual model sorption model when applied to sorption-desorption hysteresis. To overcome this, a unified dual mode sorption model was introduced that allows for hole formation during sorption. When applied to sorption kinetics, the redistribution between the two populations occurs continuously, removing the discontinuity. The dissolved population and its gradient are considerably lower throughout the sorption process, leading to a higher diffusion coefficient, on par with that for desorption.

At the two lowest activities, the diffusion coefficients for sorption and desorption are nearly the same, within error. However, at the two highest activities, the desorption diffusion coefficient remains a factor of two to three higher than the sorption value. In part, this is the result of desorption data that were taken over only half the entire concentration range, the higher half, which for diffusion coefficients that increase with concentration, will result in averaged values that are higher than those measured over the entire range, as they are in sorption. In addition, the actual nature of the concentration dependence may itself be different between sorption and desorption. Lastly, because the desorption model developed here does not allow for holes to collapse, a further redistribution between the two 
populations upon desorption may result in a lowering of those diffusion coefficients, particularly at higher activities.

The approach taken in this study to unify sorption and desorption kinetics in a glassy polymer is based on a modification of the classic dual mode model, which presumes that any depression of the glass-transition temperature due to penetrant sorption is the result of enhanced local mobility around the dissolved population with little effect on the hole population. In other words, the Langmuir capacity remains fixed (classic) or increases (with hole formation) even though the glass-transition temperature decreases with increasing concentration. Therefore, the glass-transition concentration represents the point at which chain relaxation "releases" or mobilizes the entire hole population. The concept of hole formation during sorption, as developed here, could be incorporated into other models on the basis of the dual mode construct. For example, Kamiya, et al. ${ }^{15}$ modified the classic dual mode model to allow for hole relaxation with increasing concentration, resulting in a Langmuir capacity that decreases linearly with concentration. In their model, the glass transition represents the point at which the last populated hole is mobilized. It would be interesting to test the approach here with this and other dual mode models and with other penetrantpolymer systems.

Acknowledgment. Financial support for J.G. from the University of Maryland, College Park is gratefully acknowledged.

\section{References and Notes}

(1) Elabd, Y. A.; Baschetti, M. G.; Barbari, T. A. J. Polym. Sci., Part B: Polym. Phys. 2003, 41, 2794-2807.

(2) Hong, S. U.; Barbari, T. A.; Sloan, J. M. J. Polym. Sci., Part B: Polym. Phys. 1997, 35, 1261-1267.

(3) Baschetti, M. G.; Piccinini, E.; Barbari, T. A.; Sarti, G. C. Macromolecules 2003, 36, 9574-9584.

(4) Elabd, Y. A.; Barbari, T. A. Ind. Eng. Chem. Res. 2001, 40, 3076 3084.

(5) Poling, B. E.; Prausnitz, J. M.; O'Connell, J. P. The Properties of Gases and Liquids, 5th ed.; McGraw-Hill: New York, 2001, p 638

(6) Elabd, Y. A.; Barbari, T. A. AIChE J. 2001, 47, 1255-1262.

(7) Crank, J. The Mathematics of Diffusion, 2nd ed.; Clarendon Press: Oxford, U.K., 1975; $\mathrm{p} 47$.

(8) Michaels, A. S.; Vieth, W. R.; Barrie, J. A. J. Appl. Phys. 1963, 34, $1-12$.

(9) Paul, D. R.; Yampol'skii, Yu. P. Polymeric Gas Separation Membranes; CRC Press: Boca Raton, FL, 2000; p 313.

(10) Fleming, G. K.; Koros, W. J. Macromolecules 1986, 19, 22852291.

(11) Jordan, S. M.; Koros, W. J.; Fleming, G. K. J. Membr. Sci. 1987, 30, 191-212.

(12) Berens, A. R.; Hopfenberg, H. B. J. Polym. Sci., Part B: Polym. Phys. 1979, 17, 1757-1770.

(13) Berens, A. R.; Hopfenberg, H. B. Polymer 1978, 19, 489-496.

(14) Fleming, G. K.; Koros, W. J. J. Polym. Sci., Part B: Polym. Phys. 1990, 28, 1137-1152.

(15) Kamiya, Y.; Hirose, T.; Mizoguchi, K.; Naito, Y. J. Polym. Sci., Part B: Polym. Phys. 1986, 24, 1525-1539. 